\section{Patch Repair of Anomalous Origin of the Left Main Coronary Artery From the Anterior Aortic Sinus}

\author{
Jun Li, MD, PhD, * Hao Lai, MD, PhD,* \\ Jiayu Zheng, MD, Changfa Guo, MD, PhD, \\ Jiawei Gu, MD, and Chunsheng Wang, MD
}

Department of Cardiac Surgery, Zhongshan Hospital, Fudan University, Shanghai, China

Anomalous origin of the left main coronary artery from the anterior aortic sinus is a rare but potentially lethal congenital disease. We report two adolescent patients with this anomaly who underwent surgical plasty using a saphenous vein patch, rather than unroofing or fenestration technique, as was typically done. This report suggests that surgical angioplasty can be performed safely and effectively to treat this anomaly.

(Ann Thorac Surg 2014;97:e59-61)

(C) 2014 by The Society of Thoracic Surgeons

\begin{abstract}
A nomalous origin of the left main coronary artery (LMCA) from the anterior aortic sinus is a rare but potentially lethal congenital anomaly in the young. Surgery is indicated when the diagnosis is made and there is at least one episode of cardiac event [1]. Different techniques, unroofing, and fenestration, for example, have been described previously. In this report, none of the described techniques was appropriate for our patients. Therefore, we used patch plasty technique to perform the repair.
\end{abstract}

Patient 1 was a 15-year-old girl referred to our institute for repeated episodes of exercise-induced syncope. She was asymptomatic at presentation and had normal treadmill exercise study results. An angiogram revealed an anomalous origin of the LMCA with a stenotic orifice. Patient 2 was a 14-year-old boy who developed sudden severe retrosternal chest pain, dyspnea, and diaphoresis during exercise. While being transported to hospital, he was in ventricular fibrillation and was immediately defibrillated. An electrocardiogram showed significant signs of acute myocardial ischemia, and blood tests showed elevated troponin levels. After transferring to our institute, the patient had no more symptoms at rest. A repeated electrocardiogram and multiple serum troponin test results were negative for ischemia. Multi-detector computed tomography coronary angiography (MDCTCA) was also performed in both patients and confirmed the ectopic LMCA origin arising from the anterior sinus with a hairpin-angle takeoff, and coursing between aorta and pulmonary trunk (Fig 1).

Accepted for publication Aug 27, 2013.

*JL and HL are co-first authors.

Address correspondence to Dr Wang, Department of Cardiac Surgery, Zhongshan Hospital, Fudan University, 180 Fenglin Rd, Shanghai 200032, China; e-mail: cswang@medmail.com.cn.
During operations, a transverse aortotomy was made, and the exact anatomy was explored inside the aorta. The LMCA orifice was slitlike and located just to the left of right coronary orifice adjacent to the valve commissure. The accurate course of LMCA was explored with a $1.5-\mathrm{mm}$ probe. The initial 5-8 $\mathrm{mm}$ of LMCA was located underneath the valve commissure within the aortic wall, and the remaining LMCA was identified as an extramural segment. After removing the ostial ridge, an incision was made through the cutting edge of the aorta into LMCA orifice. Subsequently, the incision was further extended longitudinally to the bifurcation of circumflex and left anterior descending artery branches. A saphenous vein was trimmed into a fusiform-shaped patch and sutured to the LMCA opening with a continuous 7-0 Prolene suture (Ethicon, Somerville, NJ). Once the patch reaches the aortic wall, a 4-0 Prolene suture was used to anastomose the patch to the wall. The remaining aortotomy was closed in the usual fashion. The onlay patch enlarges both LMCA and adjacent $10 \mathrm{~mm}$ of aortotomy and creates a funnel-shaped LMCA origination (Fig 2).

Both patients had uneventful postoperative courses and were discharged without complications after 4 days. They maintained on low-dose aspirin for 6 months postoperatively. At the first annual follow-up, both patients reported no symptoms and showed no ischemic change in exercise testing. At follow-up visit 4 years postoperatively, patient 1 remained asymptomatic with physical activities. MDCT-CA confirmed a patent augmented LMCA. An echocardiogram demonstrated a wide-open orifice with physiological flow pattern and no signs of onlay patch calcification or aneurysmal dilatation.

\section{Comment}

Anomalous origin of the LMCA from the anterior aortic sinus is a rare anomaly, with an estimated incidence of $0.02 \%$ to $0.08 \%$ according to various reports $[1,2]$. The asymptomatic patients can initially present with unexpected syncope or even sudden death [2]. Thus, diagnosis must be suspected in any young individuals with exercise-induced complaints suggestive of myocardial ischemia. MDCT-CA is crucial in establishing diagnosis. The course and the patency of the LMCA can be delineated even more precisely than using angiography.

The exact mechanism of sudden death remains unclear. Cohen and Shaw [3] postulated that compression of the anomalous LMCA between the great vessels played an important role on episodic myocardial ischemia. However, it seems impossible that a low-pressure pulmonary trunk could compress the LMCA distended by systemic blood pressure if there is no underlying disease condition causing pulmonary hypertension [2]. Generally, the LMCA is oriented at an approximately 90-degree angle from the left posterior sinus, but in this anomaly it arises from the aorta with a sharp angle and a slitlike orifice. During exercise, ostial closure and luminal narrowing of the intramural segment can occur because of acute stretching with increased tension of the aortic wall [4]. We 
Fig 1. (A, B) Anomalous LMCA that arose from anterior sinus with a hairpin-angle takeoff preoperatively in patient 2. $(C, D) A$ funnel-shaped orifice and a much better LMCA angulation postoperatively.

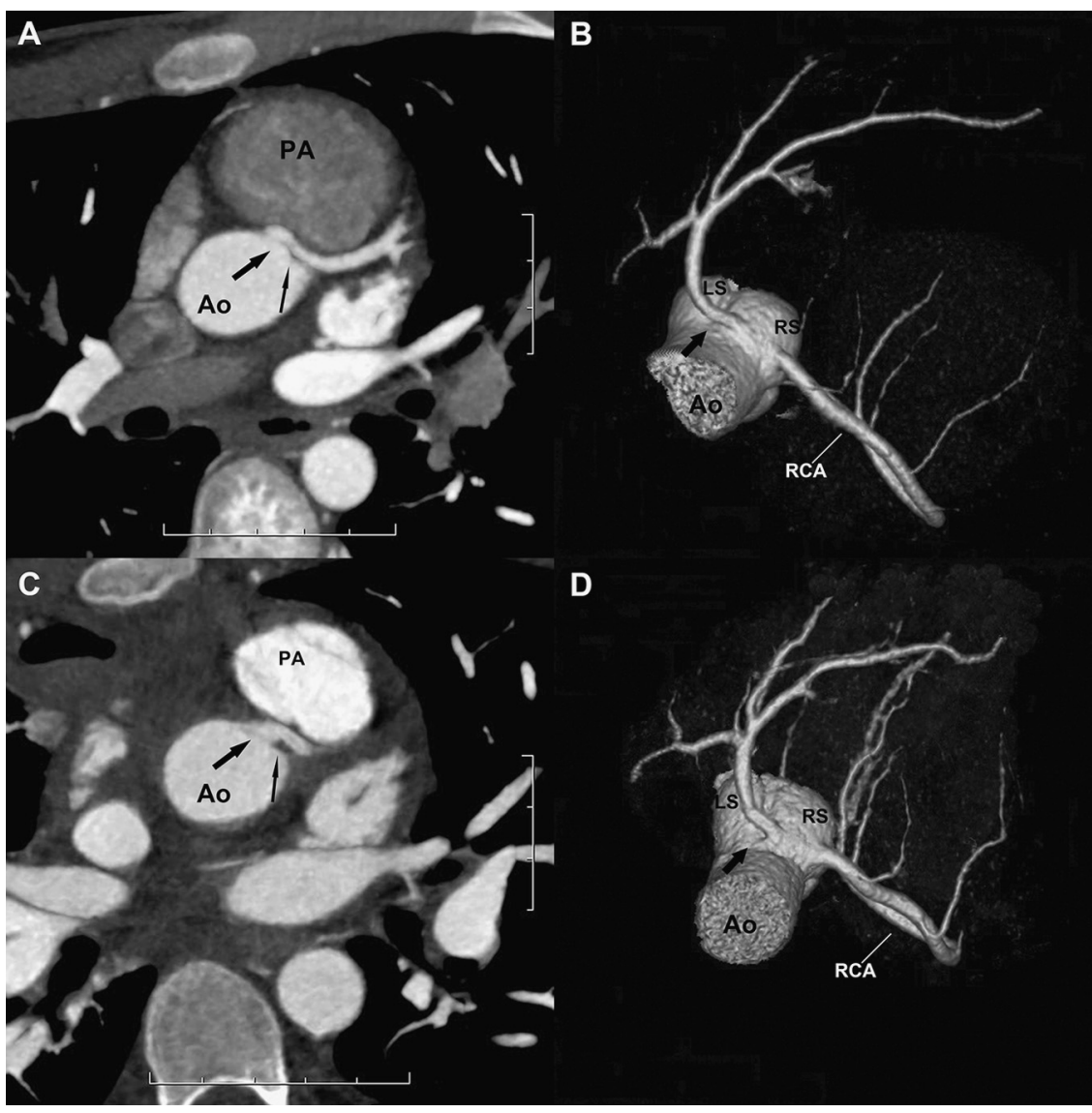

believe that the etiology of sudden death with this anomaly is more likely related to the angular alteration of the LMCA origination than to the lateral compression from the pulmonary trunk.

Several sophisticated techniques have been developed over the years and can be classified into two categories. One is appropriate for repairing intramural segment, including unroofing and fenestration [1, 5]; the other is mainly dealing with the extramural segment including orifice reimplantation and pulmonary trunk translocation $[6,7]$. Our patients were not amenable to unroofing, because the initial segment encroached on the valve commissure. As the LMCA became an extramural portion when it passed over the left posterior sinus, no adequate length of common wall could be cut out to form a widen neo-ostium. As a result, fenestration was not feasible for
Fig 2. Pulmonary trunk was retracted leftwards, and adequate exposure could be obtained (view from the surgeon). (A) An incision was made through the aorta cutting edge into LMCA orifice (black arrows). (B) A saphenous vein patch (black arrows) was used to enlarge the LMCA and created a funnel-shaped orifice. $($ Ao $=$ aorta; $L S=$ left posterior sinus; $P A=$ pulmonary artery; $R A A=$ right atrial appendage; $R S=$ anterior sinus.)

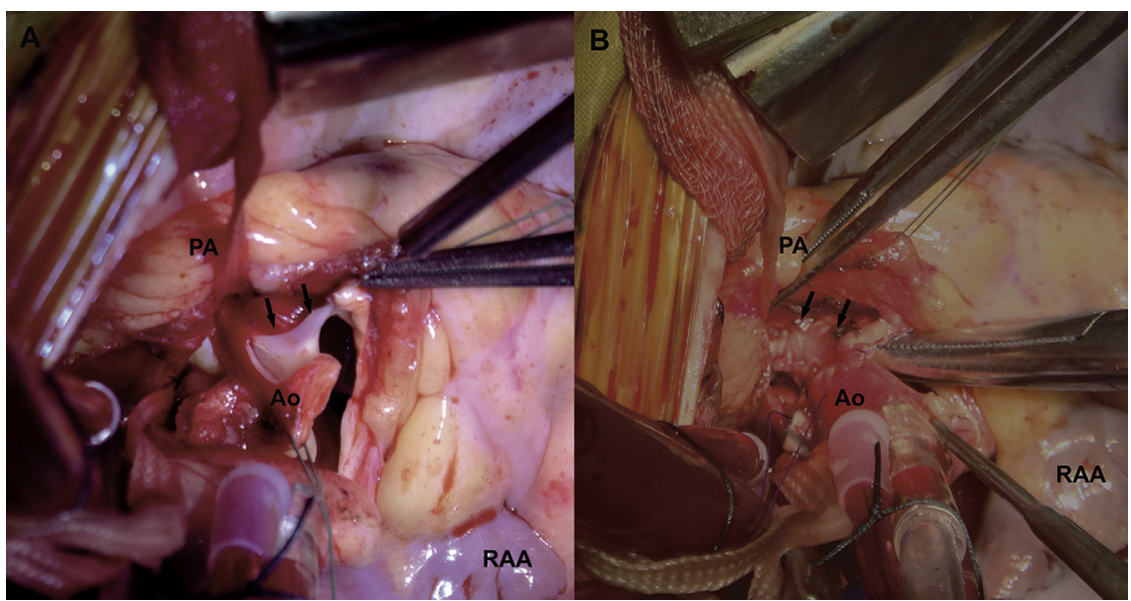


either patient. Orifice reimplantation was difficult because the anomalous LMCA could not be excised completely with a Carrel patch because of its slitlike orifice and intramural segment. Pulmonary trunk translocation, theoretically, might relieve compression of the LMCA from great vessels, but still leave the tangential origination unsolved. Our approach aimed to eliminate the slitlike orifice, tangential origination, and luminal narrowing of the intramural segment. Because of the concern of inadequate exposure of the LMCA, pulmonary trunk transection was applied in patient 1 . For patient 2, we retracted the pulmonary trunk laterally and found that this provided sufficient exposure without interfering with pulmonary artery intact.

Currently, there is no specific preference regarding selection of patch materials. We prefer the saphenous vein because it is large enough to make a funnel-shaped ostium. Furthermore, the saphenous vein is superior to the pericardium because of its potential fibrinolytic activity, which might prevent degeneration and thrombosis [8]. Although the internal mammary artery has a higher long-term patency compared with venous graft in coronary artery bypass grafting, it may be difficult to obtain an adequate size of material as an onlay patch in practice. As with pulmonary autograft, the decision should be made prudentially because of the inevitable large defect on pulmonary artery.

During postoperative follow-up, exercise testing, MDCT-CA, and echocardiogram are sufficient for asymptomatic patients. Because of the invasive nature of these methods, angiogram and intravascular ultrasound should be performed only if the patient has symptoms.

\section{References}

1. Mustafa I, Gula G, Radley-Smith R, Durrer S, Yacoub M. Anomalous origin of the left coronary artery from the anterior aortic sinus: A potential cause of sudden death. Anatomic characterization and surgical treatment. J Thorac Cardiovasc Surg 1981;82:297-300.

2. Cheitlin MD, De Castro CM, McAllister HA. Sudden death as a complication of anomalous left coronary origin from the anterior sinus of valsalva: a not-so-minor congenital anomaly. Circulation 1974;50:780-7.

3. Cohen LS, Shaw LD. Fatal myocardial infarction in an 11-yearold boy associated with a unique coronary artery anomaly. Am J Cardiol 1967;19:420-3.

4. Maron BJ. Sudden death in young athletes. N Engl J Med 2003;349:1064-75.

5. Van Son JA, Mohr FW. Modified unroofing procedure in anomalous aortic origin of left or right coronary artery. Ann Thorac Surg 1997;64:568-9.

6. Fernandes ED, Kadivar H, Hallman GL, et al. Congenital malformations of the coronary arteries: The Texas Heart Institute experience. Ann Thorac Surg 1992;54:732-40.

7. Rodefeld MD, Culbertson CB, Rosenfeld HM, Hanley FL, Thompson LD. Pulmonary artery translocation: a surgical option for complex anomalous coronary artery anatomy. Ann Thorac Surg 2001;72:2150-2.

8. Dion R, Elias B, El Khoury G, et al. Surgical angioplasty of the left main coronary artery. Eur J Cardiothorac Surg 1997;11: 857-64. 